\title{
Contents
}

\section{Multivariate Time Series Analysis}

Björn Schelter, Rainer Dahlhaus, Lutz Leistritz, Wolfram Hesse,

Bärbel Schack, Jürgen Kurths, Jens Timmer, Herbert Witte .......... 1

2 Surrogate Data - A Qualitative and Quantitative Analysis

Thomas Maiwald, Enno Mammen, Swagata Nandi, Jens Timmer ...... 41

\section{Multiscale Approximation}

Stephan Dahlke, Peter Maass, Gerd Teschke, Karsten Koch,

Dirk Lorenz, Stephan Müller, Stefan Schiffler, Andreas Stämpfli,

Herbert Thiele, Manuel Werner ......................... 75

\section{Inverse Problems and Parameter Identification in Image} Processing

Jens F. Acker, Benjamin Berkels, Kristian Bredies, Mamadou S. Diallo, Marc Droske, Christoph S. Garbe, Matthias Holschneider,

Jaroslav Hron, Claudia Kondermann, Michail Kulesh, Peter Maass,

Nadine Olischläger, Heinz-Otto Peitgen, Tobias Preusser,

Martin Rumpf, Karl Schaller, Frank Scherbaum, Stefan Turek ...

5 Analysis of Bivariate Coupling by Means of Recurrence

Christoph Bandt, Andreas Groth, Norbert Marwan, M. Carmen

Romano, Marco Thiel, Michael Rosenblum, Jürgen Kurths ........... 153

\section{Structural Adaptive Smoothing Procedures}

Jürgen Franke, Rainer Dahlhaus, Jörg Polzehl, Vladimir Spokoiny,

Gabriele Steidl, Joachim Weickert, Anatoly Berdychevski, Stephan Didas, Siana Halim, Pavel Mrázek, Suhasini Subba Rao, Joseph Tadjuidje ................................... 183 
7 Nonlinear Analysis of Multi-Dimensional Signals

Christoph S. Garbe, Kai Krajsek, Pavel Pavlov, Björn Andres, Matthias Mühlich, Ingo Stuke, Cicero Mota, Martin Böhme, Martin Haker, Tobias Schuchert, Hanno Scharr, Til Aach, Erhardt Barth,

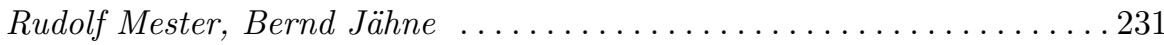

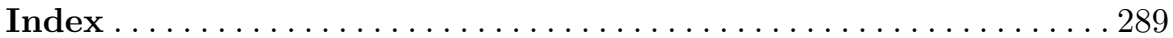

